\title{
Inhibition of Drp1-dependent mitochondrial fragmentation and apoptosis by a polypeptide antagonist of calcineurin
}

\author{
GM Cereghetti ${ }^{1}$, V Costa ${ }^{1}$ and L Scorrano*,1,2
}

During apoptosis, mitochondria lose their membrane potential and undergo fragmentation around the time of release of cytochrome $c$. Apoptotic fission is at least in part sustained by the translocation of dynamin-related protein 1 (Drp1), normally located in the cytosol, to mitochondria. This process depends on dephosphorylation of Drp1 by the phosphatase calcineurin. Here, we report the identification of a novel inhibitor of this process. A polypeptide (PPD1) from the immunophilin FKBP52 inhibits calcineurin activation triggered by mitochondrial dysfunction. PPD1 blocks Drp1 translocation to mitochondria and fragmentation of the organelle. PPD1 delays apoptosis by intrinsic stimuli by preventing fragmentation and release of cytochrome $c$. Cells expressing PPD1 display enhanced clonogenic ability after exposure to staurosporine. A genetic analysis revealed that the activity of PPD1 is independent of the BH3-only protein BAD, another target of calcineurin during apoptosis, and is not additive to inhibition of Drp1. Thus, PPD1 is a novel inhibitor of apoptosis that elucidates the function of calcineurindependent mitochondrial fragmentation in the amplification of cell death.

Cell Death and Differentiation (2010) 17, 1785-1794; doi:10.1038/cdd.2010.61; published online 21 May 2010

Mitochondria are important organelles in the regulation of apoptosis. They amplify the intrinsic pathway by releasing protein cofactors that are essential for the activation of effector caspases (such as cytochrome $c$ ) and for the direct condensation of chromatin (such as AIF). ${ }^{1}$ This release is the consequence of permeabilization of the outer membrane of the organelle and it is controlled by members of the $\mathrm{Bcl}-2$ family. In a widely accepted model, the subset of 'BH3-only' Bcl-2-family members senses the 'private' death stimuli, transferring it to mitochondria whose outer membrane is permeabilized through the 'multidomain' proapoptotic members. The action of $\mathrm{BH} 3-$ only proteins is counteracted by antiapoptotic members that prevent activation of the multidomains in the mitochondrial membrane by soaking the BH3-only members. ${ }^{2,3}$ Thus, in this model, the only mitochondrial event that has an important function in the progression of the apoptotic cascade is the loss of the controlled permeability of the outer membrane of the organelle. One of the basic tenets of apoptosis, that mitochondrial shape is unchanged, has been recently challenged, thanks to the development of more refined techniques of investigation of mitochondrial shape and ultrastructure. A new consensus developed that mitochondria undergo massive fragmentation around the time of their outer membrane permeabilization, ${ }^{4,5}$ and that the complete release of cytochrome $c$ is accompanied by ultrastructural changes collectively called cristae remodeling. ${ }^{6}$
Mitochondrial shape is determined by the equilibrium between continuously ongoing fusion and fission processes. In mammalian cells, a core machinery of 'mitochondriashaping' proteins exists that impinges on fusion or fission of the organelle. Mitochondrial fusion depends on the outer membrane mitofusins (Mfn) 1 and 2 and on the inner membrane Optic Atrophy 1 (Opa1). ${ }^{7}$ These proteins are large dynamin-related GTPases that display pleiotropic functions: for example, Opa1 regulates biogenesis and remodeling of the cristae independently of its function in fusion, ${ }^{8,9}$ whereas Mfn2 tethers mitochondria to the endoplasmic reticulum. ${ }^{10}$ Mitochondrial fission depends on the translocation of cytoplasmic dynamin-related protein 1 (Drp1) to mitochondria, in which it binds to its adaptor in the outer membrane, Fis1, oligomerizes, and constricts the organelle, ultimately leading to its fission. ${ }^{11,12}$ Dephosphorylation of Drp1 by the cytosolic $\mathrm{Ca}^{2+}$-dependent phosphatase calcineurin regulates its subcellular localization and promotes its translocation to mitochondria. ${ }^{13,14}$

The molecular knowledge of the players in mitochondrial shape regulation stimulated intense research in the last few years on their function in cell death. In particular, a dominantnegative mutant of Drp1, in which substitution of lysine 38 for an alanine in the GTPase domain disrupted its enzymatic activity, prevents fragmentation of the mitochondrial network, mitochondrial membrane depolarization, cytochrome $c$

\footnotetext{
${ }^{1}$ Department of Cellular Physiology and Metabolism, University of Geneva, 1 Rue M. Servet, Geneva, Switzerland and ${ }^{2}$ Dulbecco-Telethon Institute, Venetian Institute of Molecular Medicine, Via Orus 2, Padova 35129, Italy

${ }^{*}$ Corresponding author: L Scorrano, Department of Cell Physiology and Metabolism, University of Geneva, Rue M. Servet, 1, Geneva CH-1206, Switzerland. Tel: + 41223795 235; Fax: + 41223795 260; E-mail: luca.scorrano@ unige.ch

Keywords: mitochondria; fission; calcineurin; Drp1

Abbreviations: Drp1, dynamin-related protein 1; PPD1, FKBP52 peptidylprolyl isomerase domain 1; Mfn, mitofusin; Opa1, optic atrophy 1; CsA, cyclosporine A; PTP, permeability transition pore; FKBP52, FK506-binding protein 52; CnA, calcineurin; CypA, cyclophilin A; ArA, arachidonic acid; FCCP, carbonylcyanide p-trifluoromethoxyphenyl-hydrazone; mtRFP, mitochondrially targeted dsRED; YFP, yellow fluorescent protein; MEFs, mouse embryonic fibroblasts; GFP, green fluorescent protein; Sts, staurosporine; VP16, etoposide; TG, thapsigargin

Received 05.11.09; revised 16.3.10; accepted 14.4.10; Edited by R Youle; published online 21.5.10
} 
release, and cell death induced by staurosporine, placing Drp1 and mitochondrial fragmentation early in apoptosis. ${ }^{4}$ Further, Drp1 was found to accumulate at mitochondrial fission sites together with Bax and Mfn2. ${ }^{15}$ Dominant-negative Drp1 was shown not to impede Bax translocation to mitochondria, implying that Bax translocation alone is not sufficient for cell death induction. ${ }^{9,15-17}$ Interestingly, downregulation of Drp1 by shRNA showed that even if inhibiting cytochrome $c$ release, absence of Drp1 was not sufficient to prevent release of other proapoptotic factors from mitochondria on induction of cell death, suggesting that mitochondrial subcompartimentalization of cytochrome $c$ stores might be altered by Drp1. ${ }^{16,18}$ Accordingly, remodeling of the cristae can be also blocked by a dominant-negative mutant of Drp1 during apoptosis induced by the $\mathrm{BH} 3$-only protein $\mathrm{BIK},{ }^{19}$ and in $\mathrm{Drp1}^{-/-}$cells, cytochrome $c$ release in response to apoptotic stimuli is delayed, whereas release of Smac/DIABLO is unaffected. ${ }^{20}$ Recently, Nunnari and coworkers found that mdivi-1, a specific chemical inhibitor of Drp1 GTPase activity, prevents both mitochondrial fission and Bax-mediated mitochondrial outer membrane permeabilization. ${ }^{21}$ Although there is mounting evidence that mitochondrial fission participates in apoptosis, recent reports questioned whether genetic maneuvers aimed at upregulating mitochondrial fusion are cytoprotective, ${ }^{22}$ leaving the question of whether mitochondrial fragmentation is a decision step toward cell death.

We recently showed that Drp1 interacts with the cytoplasmic peptidylprolyl isomerase cyclophilin A (CypA) in the cytosol. This interaction is lost on induction of mitochondrial fragmentation triggered by depolarization of the organelle and calcineurin activation. ${ }^{14}$ On dephosphorylation at the Ser 637 residue by calcineurin, Drp1 translocates to mitochondria causing their fission. This calcineurin loop in principle could provide a target to manipulate apoptotic mitochondrial fission. However, two problems should be taken into account when addressing this possibility. First, compounds that inhibit calcineurin, such as cyclosporine $A(\mathrm{CsA})$, also act on the permeability transition pore (PTP), whose function in at least certain paradigms of cell death is well established. Second, it has been shown that CypA is associated with dynamitin, a component of the dynein-associated dynactin molecular motor complex responsible for retrograde protein transport along the microtubules network, and this association can be blocked by $\mathrm{CsA}^{23}$ Dynein has also been implied in the translocation of Drp1 to mitochondria, further complicating the picture. ${ }^{24}$ This raises the question of whether inhibitors of calcineurin affect translocation of Drp1 to mitochondria specifically by blocking dephosphorylation of Drp1, or whether they act indirectly, by interfering with the interplay between Drp1 and dynein. In an attempt to find an answer to these questions, our attention was caught by a peptide corresponding to the peptidylprolyl isomerase domain of immunophilin FK506-binding protein 52 (FKBP52; residues ranging from glycine 32 to lysine 138), which is able to inhibit association between CypA and dynein. ${ }^{23,25}$ This peptide represents a useful tool to address these questions. Here, we show that expression of the FKBP52 peptide (PPD1) prevents Drp1 interaction with CypA and calcineurin, but does not dissociate Drp1 from dynein. PPD1 still inhibits mitochondrial fragmentation by blocking calcineurin activity and preventing recruitment of Drp1 to mitochondria. In cells expressing PPD1, translocation of Bax and release of Smac/DIABLO are unaffected, whereas mitochondrial fragmentation, cytochrome $c$ release, and cell death induced by different apoptotic stimuli are prevented. The action of PPD1 is independent of Bad, another important target of calcineurin during apoptosis, and is not additive to blockage of Drp1. Thus, PPD1 further supports a function for calcineurin-mediated mitochondrial fragmentation during apoptosis and it unveils a novel potential site of action to intervene in therapy of pathological conditions in which excess apoptosis should be blocked.

\section{Results}

PPD1 inhibits depolarization-induced mitochondrial fragmentation. Mitochondrial depolarization triggers calcineurin activation, dephosphorylation of Drp1, and its translocation to mitochondria, both blocked by the calcineurin inhibitors FK506 and CsA. In fact, Drp1 interacts with calcineurin and with CypA and it is largely associated to microtubules. On mitochondrial dysfunction, Drp1 leaves this complex and the microtubules translocating to mitochondria. ${ }^{14}$ Interestingly, the microtubule-anchored dynein motor complex regulates Drp1 translocation to mitochondria $^{24}$ and CypA was shown to act as a mediator of binding of proteins to the dynein motor complex. ${ }^{23}$ Thus, the inhibitory effect of FK506 and CsA might depend on their ability to displace Drp1 from dynein. To test this possibility, we turned to PPD1, a peptide corresponding to the peptidylprolyl isomerase domain of immunophilin FK506binding protein 52 (aa. 32-138) that displaces CypA from dynein. We therefore checked whether PPD1 had any effect on mitochondrial depolarization or fragmentation. Expression of PPD1 did not prevent mitochondrial depolarization induced by the lipid arachidonic acid (ArA), as shown by real-time imaging of the mitochondrial fluorescence of the potentiometric dye tetramethyl rhodamine methylester (TMRM) loaded in HeLa cells cotransfected with GFP and PPD1 or an empty vector (Figure 1a). Similarly, expression of PPD1 did not affect depolarization by the protonophore carbonylcyanide p-trifluoromethoxyphenyl-hydrazone (FCCP). ArA and FCCP induce rapid fragmentation of HeLa mitochondria, causing the transition from network to individual organelles in approximately $10 \mathrm{~min}$. Of note, PPD1 greatly delayed depolarization-induced, Drp1dependent mitochondrial fragmentation, even if it had no effect on mitochondrial depolarization (Figure $1 \mathrm{~b}$ and $\mathrm{c}$ ).

PPD1 displaces CypA from Drp1, inhibiting calcineurin activation and Drp1 dephosphorylation. PPD1 could, therefore, represent a novel inhibitor of mitochondrial fragmentation acting by displacing Drp1 from dynein. To test this hypothesis, we immunoprecipitated Drp1 in HeLa cells and verified how PPD1 and mitochondrial dysfunction modulated its association with dynein. Western blot analysis of the co-immunoprecipitated material showed that interaction of Drp1 with CypA was diminished by expression of PPD1 (Figure 2a). On the contrary, interaction of Drp1 with dynein was not influenced by the 
a

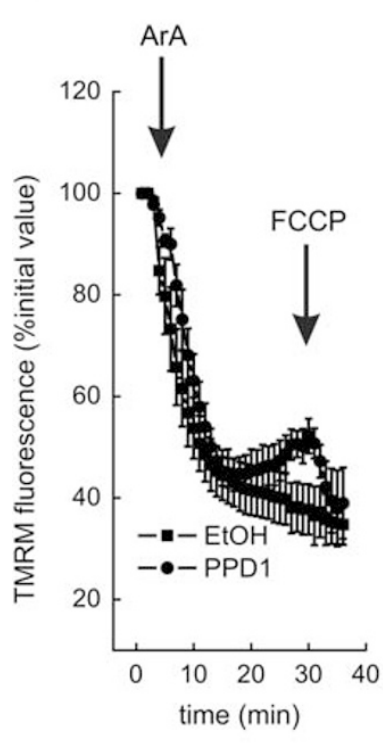

b
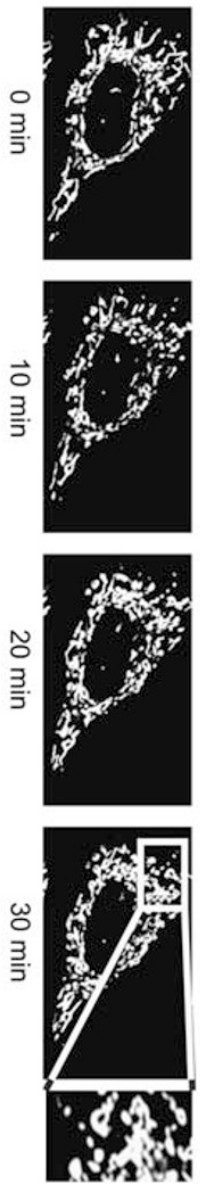

FCCP

PPD1
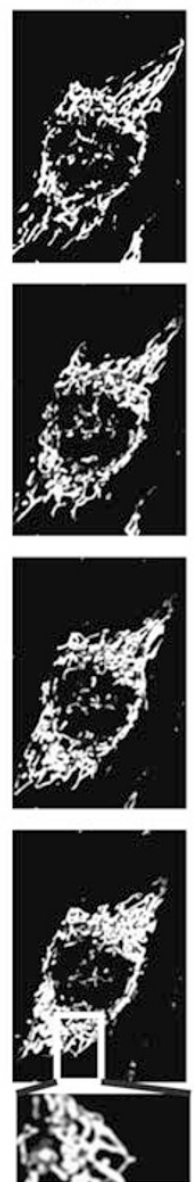

ArA
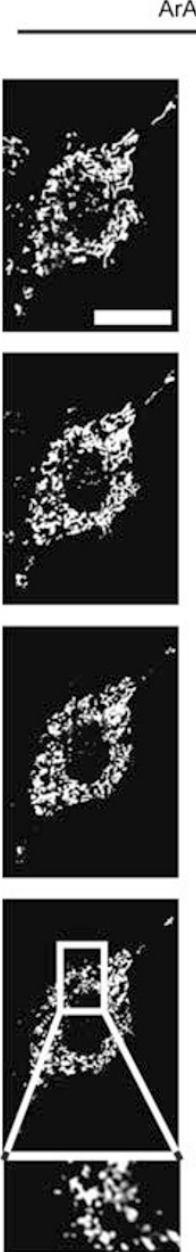
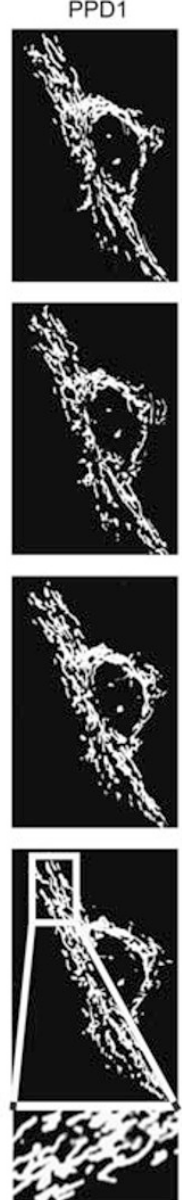

C

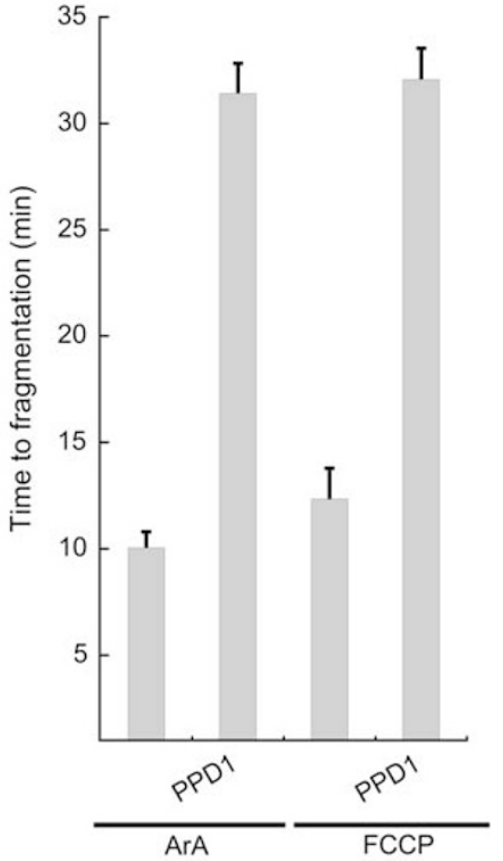

Figure 1 PPD1 delays mitochondrial fragmentation induced by mitochondrial depolarization. (a) Changes in mitochondrial fluorescence of TMRM. HeLa cells loaded with TMRM were treated where indicated (arrows) with $20 \mu \mathrm{M}$ ArA or $2 \mu \mathrm{M}$ FCCP. Fluorescence intensities were normalized to the initial value for comparative reasons. Data represent mean \pm S.E. of four independent experiments. (b) Representative frames acquired at indicated times from real-time confocal imaging of HeLa cells cotransfected with mtRFP and PPD1 or empty vector. At $t=3 \mathrm{~min}$, cells were treated where indicated with $20 \mu \mathrm{M}$ ArA or $2 \mu \mathrm{M} \mathrm{FCCP.} \mathrm{Bar,} 20 \mu \mathrm{m}$. The boxed areas of the last frames are enlarged $\times 3$. (c) Morphometric analysis performed as described (38) of mitochondrial shape changes in HeLa cells treated exactly as described in (b). Data represent mean \pm S.E. of eight different experiments ( $n=20$ cells per condition in each experiment)

presence of the peptide, indicating that interaction of Drp1 with the dynein motor complex is not mediated by the immunophilin (Figure 2). Although association of Drp1 with CypA was completely lost on induction of mitochondrial fragmentation by $\operatorname{ArA}$, interaction of the protein with dynein persisted. Thus, modulation of binding to dynein could not account for the inhibitory effect of PPD1 on mitochondrial fragmentation. Interestingly, the interaction between calcineurin and Drp1 was disrupted in the presence of PPD1 (Figure 2a), prompting us to test whether the activity of the phosphatase was also affected by PPD1. A specific assay indicated that after mitochondrial dysfunction by both ArA and FCCP activity of calcineurin increases and that this is completely abrogated in cells expressing PPD1 (Figure 2b). Thus, PPD1 has two important effects: it acts as a calcineurin inhibitor that is genetically encoded and, therefore, more stable as opposed to the small pharmacological inhibitors that are metabolized such as CsA and FK506, and it displaces calcineurin from Drp1.
Accordingly, expression of PPD1 increased the phosphorylation of Ser637 of Drp1 and inhibited the dephosphorylation triggered by ArA, as indicated by specific immunoblotting (Figure $2 \mathrm{c}$ ). These results prompted us to investigate whether under steady state conditions, mitochondrial elongation was enhanced in cells expressing PPD1. Interestingly, expression of PPD1 almost doubled the length of mitochondria (Figure $3 a$ and quantification in Figure $3 b$ ). In conclusion, our results indicate that PPD1 increases the phosphorylation of Drp1 by dissociating it from calcineurin, whose activity is also inhibited in cells expressing the peptide. As a result, mitochondria in PPD1 expressing cells are more elongated.

PPD1 inhibits cell death at the mitochondrial level. We next verified whether PPD1 inhibited the translocation of Drp1 to dysfunctional mitochondria, dictated by the phosphorylation status of the dynamin-like protein. To this end, we monitored mitochondrial association of endogenous 
a

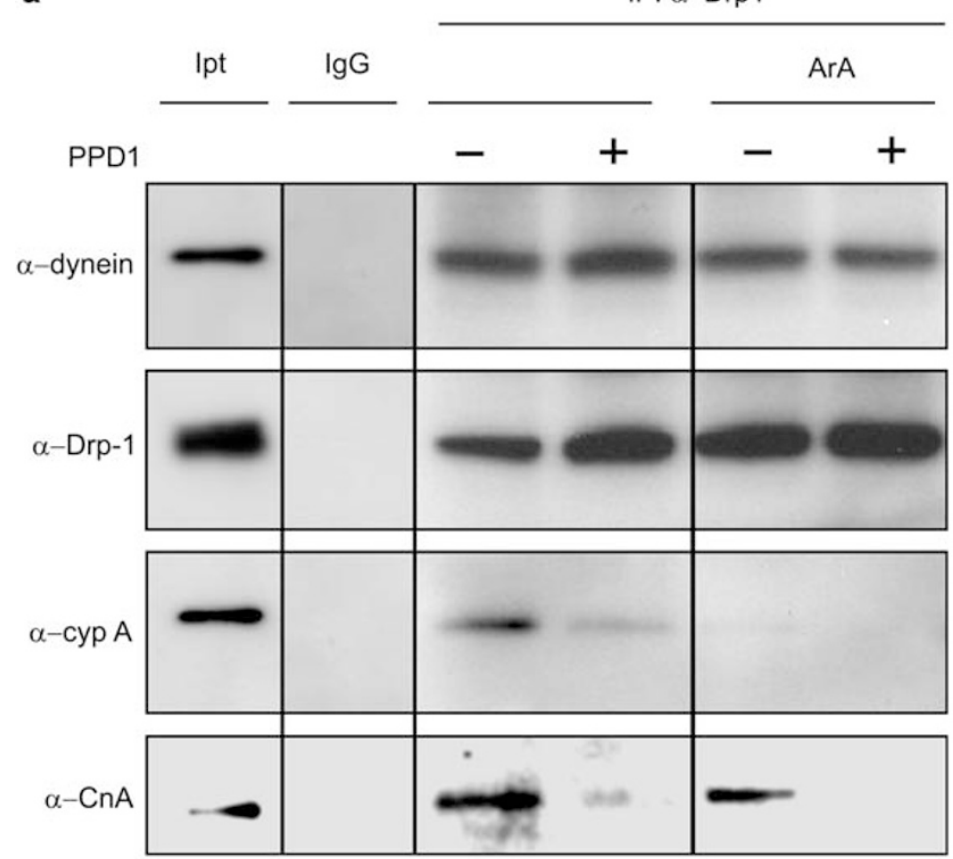

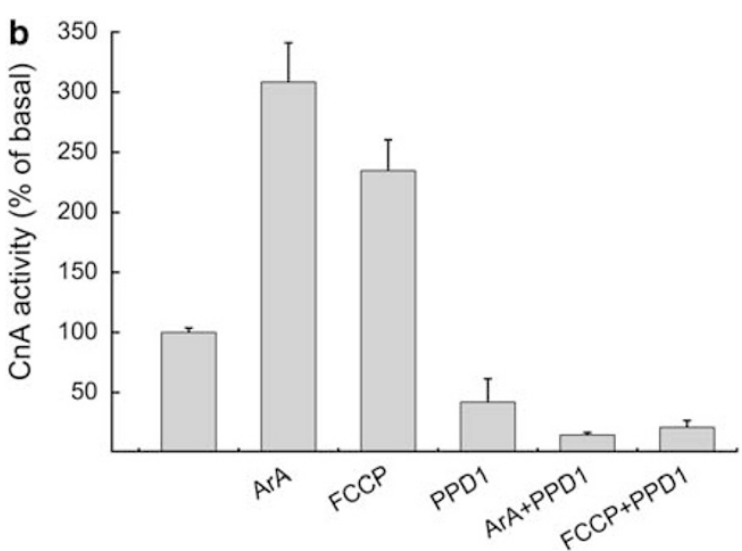

C

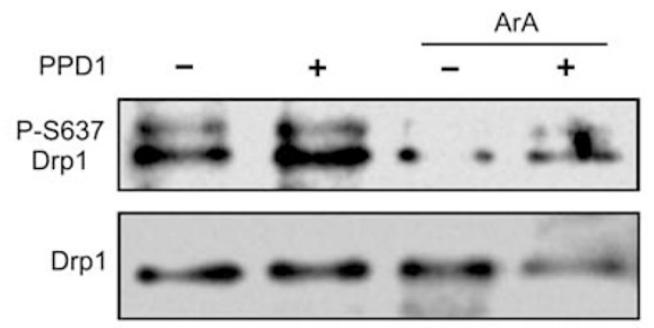

Figure 2 PPD1 displaces Drp1 from CypA and inhibits calcineurin activation and Drp1 dephosphorylation induced by mitochondrial dysfunction. (a) HeLa cells transfected as indicated were lysed and $200 \mu \mathrm{g}$ of protein were immunoprecipitated using the indicated antibody (at a 1:50 dilution). Immuno- and co-immunoprecipitated proteins from $100 \mu \mathrm{g}$ lysate were separated by SDS-PAGE and immunoblotted using the indicated antibodies. Where indicated, cells were treated with ArA ( $20 \mu \mathrm{M}, 20 \mathrm{~min}$ at RT). (b) Calcineurin activity was measured in cytosolic extracts of HeLa cells transfected with PPD1 or empty vector and treated where indicated for 15 min with $20 \mu \mathrm{M} \mathrm{ArA} \mathrm{or} 2 \mu \mathrm{M}$ FCCP. Data are compared with the activity of untreated cells set as $100 \%$ and represent mean \pm S.E. of four independent experiments. (c) HeLa cells transfected as indicated were treated with $\operatorname{ArA~(20\mu M,20~min~at~RT)~where~indicated,~lysed,~and~equal~amounts~of~proteins~(50~} \mu \mathrm{g})$ were separated by SDS-PAGE and immunoblotted using the indicated antibodies

Drp1 (Figure 3c) and of a Drp1-YFP chimera (Figure 3d) co-expressed in HeLa cells with a mitochondrially targeted dsRED (mtRFP). After mitochondrial depolarization induced by ArA or FCCP, endogenous as well as Drp1-YFP readily translocated to mitochondria and this process was inhibited by contransfection with PPD1 (Figure 3c and d). Thus, PPD1 blocks association of Drp1 with dysfunctional mitochondria. This suggests that PPD1 could interfere with apoptotic settings in which the fragmentation of mitochondria is essential for the progression of the cascade. To this end, we tested whether expression of PPD1 delayed the kinetic of apoptosis by a panoply of intrinsic inducers acting through different mechanisms. Expression of PPD1 was able to delay death by staurosporine, etoposide, and thapsigargin, three intrinsic stimuli that recruit the mitochondrial pathway of apoptosis controlled by $\mathrm{Bax}$ and $\mathrm{Bak}$ at the mitochondrial level, as well as by the pro-oxidant $\mathrm{H}_{2} \mathrm{O}_{2}$ that recruits mitochondria through the so-called 'ER gateway' similarly controlled by the two multidomain proapoptotics ${ }^{26}$ (Figure 4). Thus, PPD1 delays apoptosis by stimuli that recruit mitochondria, irrespective of their initial mechanism of action, provided that they all cause mitochondrial fragmentation. ${ }^{4,9,27}$ We further verified whether this was associated with an inhibition of mitochondrial morphological changes induced by apoptotic stimulation. We therefore followed in real time the changes in mitochondrial shape in HeLa cells by imaging the fluorescence of expressed mtRFP. On treatment with staurosporine, mitochondria underwent the known cycle of initial elongation followed by fragmentation that becomes apparent $2 \mathrm{~h}$ after induction of apoptosis. Co-expression of PPD1 with mtRFP prevented fragmentation induced by staurosporine (Figure 5a). However, PPD1 did not interfere with the translocation of Bax to the mitochondria, which occurs around the time of their fragmentation (Supplementary Figure S1) ${ }^{15}$ We turned to analyze whether PPD1 was also capable of inhibiting release of apoptogenic factors from mitochondria, by evaluating their subcellular distribution using an immunofluorescence approach ${ }^{28}$ and found that PPD1 also prevented the release of cytochrome $c$ (Figure $5 \mathrm{~b}$ and $\mathrm{c}$ ), but was not able to retain Smac/DIABLO in the intermembrane space (Supplementary Figure S2). We finally set to test whether PPD1 just delayed death, or whether it exerted a real cytoprotective effect. We measured the intrinsic ability of the survived cells to replicate and form colonies, an ultimate sign that cells have completely survived the apoptotic attack. To this end, HeLa cells were cotransfected with GFP and PPD1 (or its empty vector), sorted, plated, and treated with staurosporine. After $5 \mathrm{~h}$, cells were collected and re-plated with fresh medium devoid of apoptotic inducer. A count of individual colonies produced by the re-plated cells showed that cells expressing PPD1 were able to survive and generate new colonies despite the treatment with staurosporine (Figure $5 d$ and e). Taken together, these data indicate that PPD1 is capable of preventing cell death induced by stimuli that trigger mitochondrial permeabilization. 
a
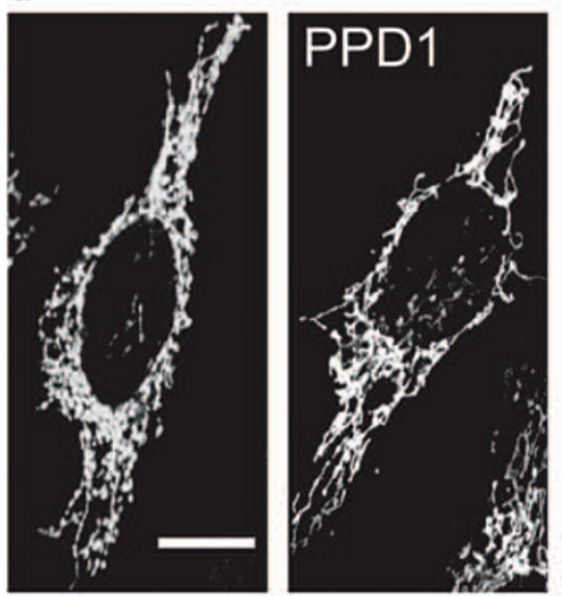

d
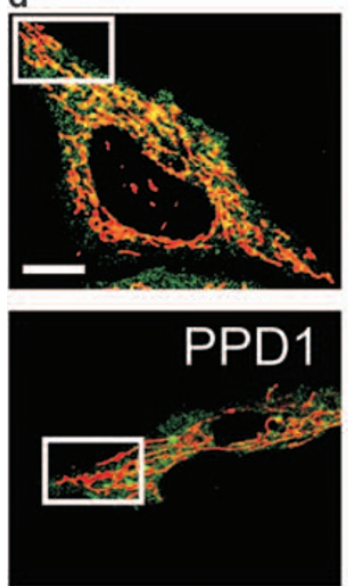

b

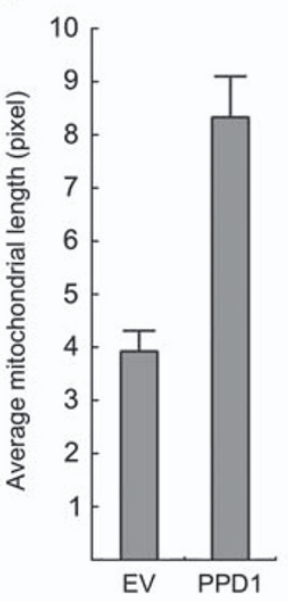

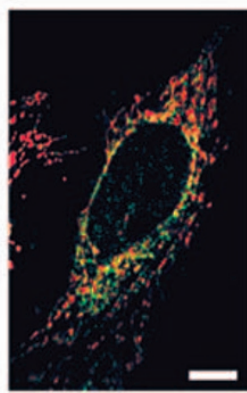

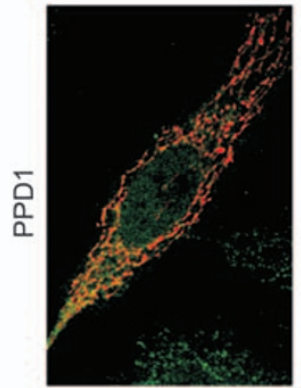

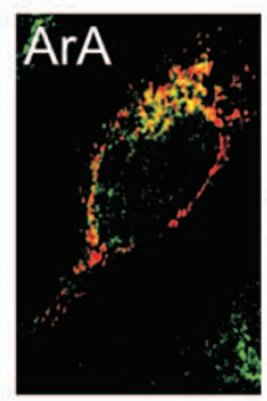

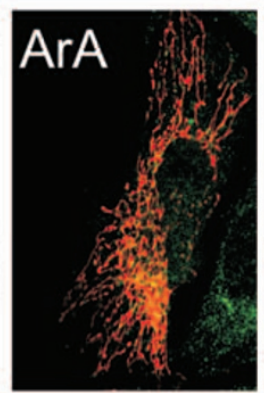

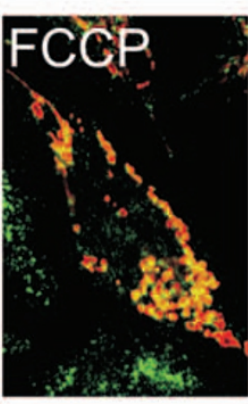

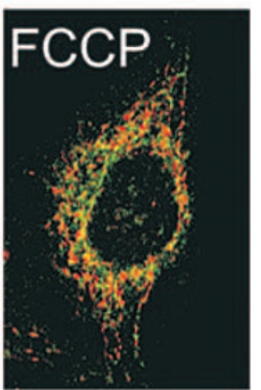

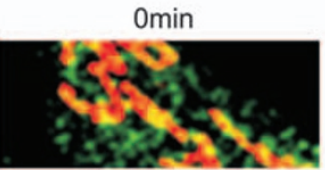
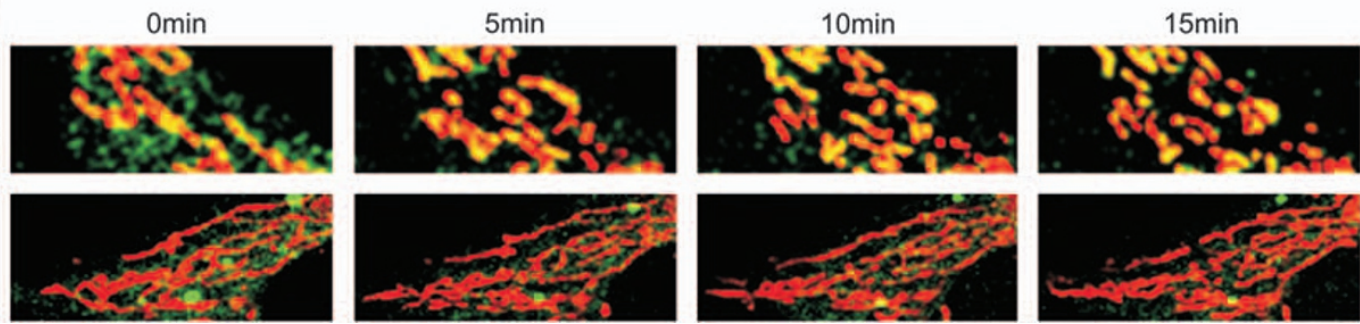

Figure 3 PPD1 elongates mitochondria and inhibits Drp1 translocation to mitochondria on dysfunction. (a) Representative confocal images of HeLa cells cotransfected with mtRFP and the indicated plasmid. Bar, $15 \mu \mathrm{m}$ (b) quantitative analysis of mitochondrial length. Experiments were as in (a) and data represent mean \pm S.E. of three independent experiments ( $n=30$ mitochondria from 10 different cells per experiment). (c) Hela cells cotransfected with mtRFP and the indicated plasmid were treated when indicated with ArA (20 $\mu \mathrm{M}, 20$ min at RT) or FCCP $(2 \mu \mathrm{M}, 20$ min at RT), fixed and immunostained for Drp1. Bar, $10 \mu \mathrm{m}$. (d) HeLa cells were cotransfected with Drp1-YFP, mtRFP, and the indicated plasmid, treated with $\operatorname{ArA}(20 \mu \mathrm{M})$ and imaged for $20 \mathrm{~min}$. The boxed areas are sixfold magnified in the image sequence. Bar, $10 \mu \mathrm{m}$

PPD1 inhibits cell death through the Drp1 pathway. Calcineurin affects a plethora of important cellular processes. For example, it dephosphorylates the proapoptotic Bcl-family protein BAD to induce its migration from the cytosol to mitochondria, leading to cell death. ${ }^{29}$ Thus, we wished to ascertain whether PPD1 protects from cell death specifically by blocking the Drp1 pathway. To this end, we first had to exclude that the effects of PPD1 were due to its ability to block dephosphorylation of BAD. We, therefore, turned to a genetic approach, comparing the ability of PPD1 to block apoptosis by staurosporine in wt and $\mathrm{Bad}^{-1-}$ mouse embryonic fibroblasts (MEFs). As expected, apoptosis by staurosporine was reduced in $\mathrm{Bad}^{-1-} \mathrm{MEFs}$ as compared with their wt counterparts (Figure 6). Expression of PPD1 was able to protect from apoptosis induced by staurosporine also in MEFs, irrespective of the presence of $B A D$. Thus, $B A D$ is dispensable for the antiapoptotic action of PPD1. We then set out to investigate whether the effect of PPD1 was mediated by Drp1. To this end, we verified whether the effect of PPD1 was additive to that of blocking
Drp1. Expression of a dominant-negative Drp1 mutant $\left(\right.$ Drp $\left.^{\mathrm{K} 38 \mathrm{~A}}\right)$ was capable of delaying death by staurosporine, such as PPD1. Of note, co-expression of PPD1 with $\mathrm{Drp}_{1}^{\mathrm{K} 38 \mathrm{~A}}$ did not result in an additional protection, indicating that they act through the same pathway (Figure 6). In conclusion, our data indicate that the inhibition granted by PPD1 is not mediated by BAD and depends on the calcineurin-Drp1 axis.

\section{Discussion}

In this study, we show that inhibition of the calcineurin-Drp1 axis by the peptide PPD1, corresponding to the peptidylprolyl isomerase domain of FKBP52, inhibits apoptosis. This is supported by a number of findings: (i) expression of PPD1 inhibits activation of calcineurin after mitochondrial dysfunction; (ii) PPD1 does not detach Drp1 from dynein, ruling out a possible alternative mechanism of inhibition of fragmentation; (iii) PPD1 blocks mitochondrial fragmentation, release of cytochrome $c$, and apoptosis by a number of intrinsic cell 

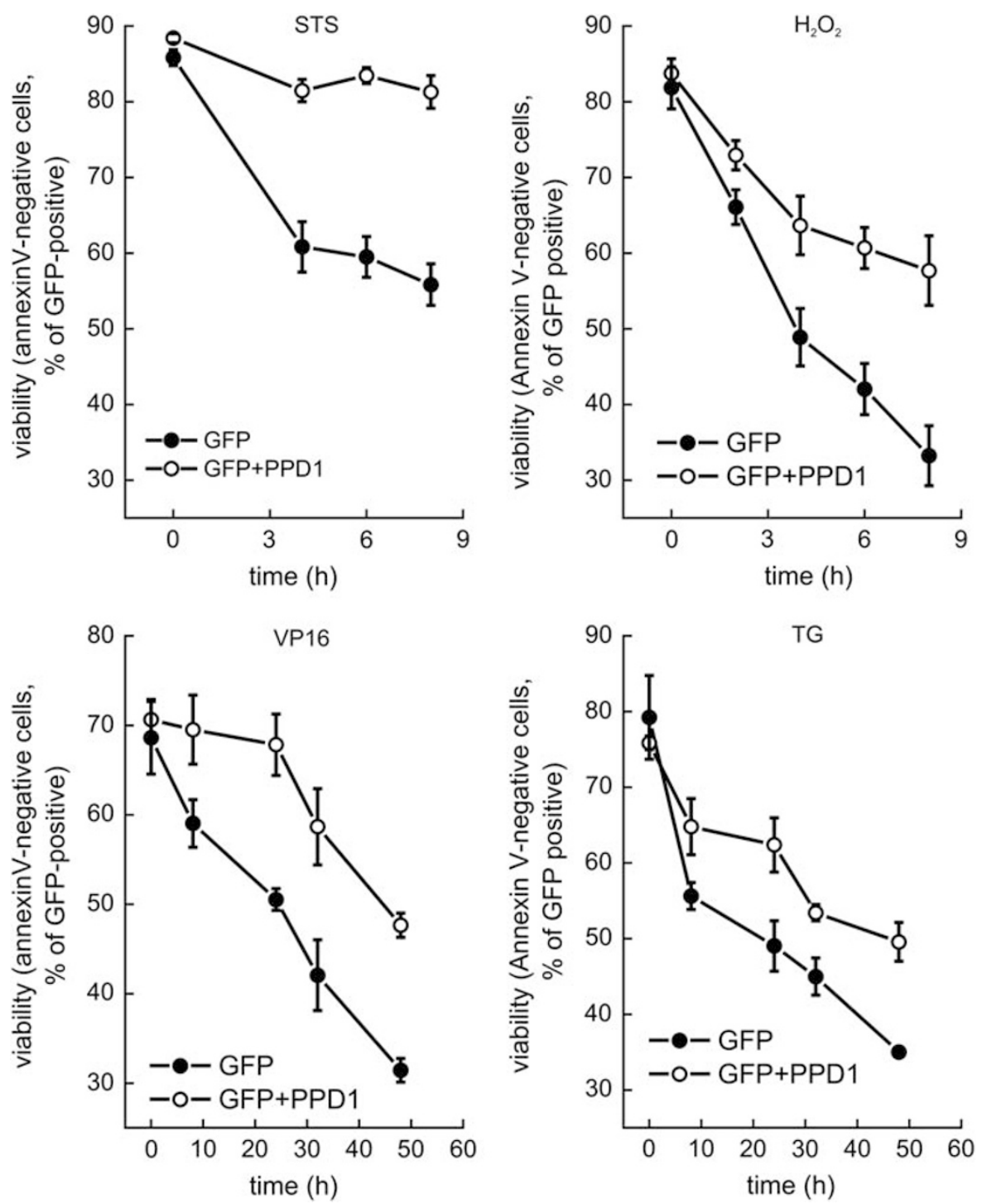

Figure 4 PPD1 prevents cell death. HeLa cells cotransfected with pEGFP and PPD1 or empty vector were treated for the indicated time with staurosporine $(2 \mu \mathrm{M})$, etoposide $(2 \mu \mathrm{M})$, hydrogen peroxide $(1 \mathrm{mM})$ or thapsigargin $(2 \mu \mathrm{M})$, collected and stained with Annexin-V. Viability was analyzed by flow cytometry as the percentage of Annexin-V-positive events in the GFP-positive population. Data represent mean \pm S.E. of five independent experiments

death stimuli, and promotes survival of cells treated with staurosporine; and (iv) a genetic analysis reveals that inhibition of cell death by PPD1 does not require BAD, another target of calcineurin during apoptosis, and is not additive to inhibition of Drp1.

In cells expressing PPD1, the interaction between Drp1 and CypA and calcineurin is disrupted, whereas that between Drp1 and dynein, the main component of the microtubules motor complex, is retained. Indeed, this peptide prevents association of immunophilin FKBP52 with dynein; ${ }^{23}$ the ability of the peptide to also blunt binding of another immunophilin, CypA, to Drp1 suggests that the interaction between the immunophilin and Drp1 involves formation of a ternary complex between Drp1, CypA, and dynein. In addition, Drp1 association with the dynein motor complex is not disrupted by induction of mitochondrial fragmentation with ArA, supporting a function for the dynein motor complex as the carrier of Drp1 to mitochondria as earlier suggested. ${ }^{24}$ The important proapoptotic molecule Bim is also associated with the dynein motor complex and this association is lost during induction of apoptosis, ${ }^{30}$ suggesting scenarios of interplay between the apoptotic and the mitochondria-shaping machineries. Our data indicate that PPD1 inhibits calcineurin. As Drp1 interacts with both CypA and calcineurin, ${ }^{14}$ we could figure out that the ability of the peptide to inhibit calcineurin is reinforced by the fact that the proteins meet on microtubules and calcineurin is sequestered and inactivated by the CypA-PPD1 complex.

PPD1 is an efficient inhibitor of mitochondrial fragmentation induced by mitochondrial depolarization. It also blocks fragmentation induced by apoptotic stimuli, such as staurosporine. Even if the function of mitochondrial fragmentation in cell death remains controversial, this finding prompted us to 

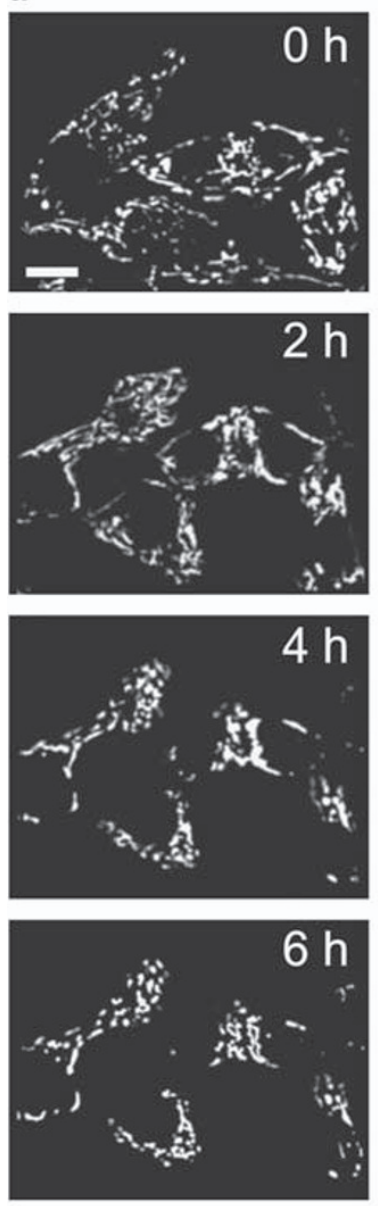

PPD1
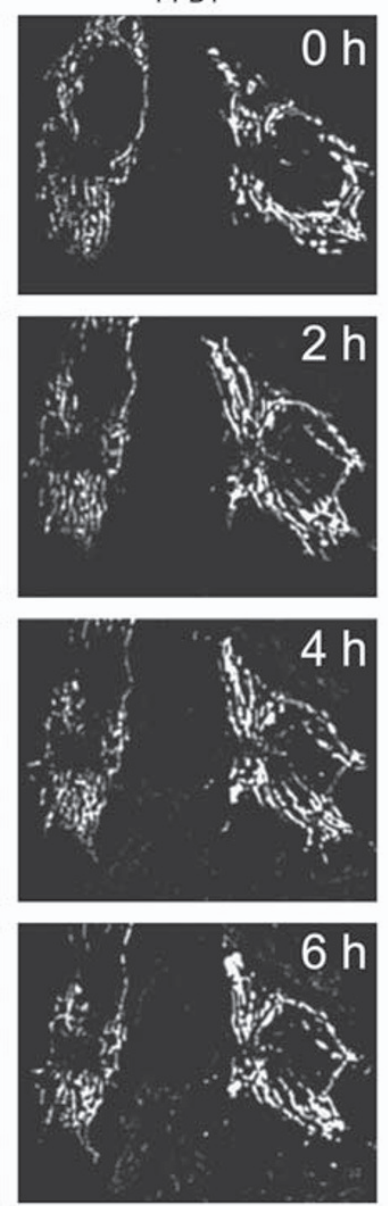

b
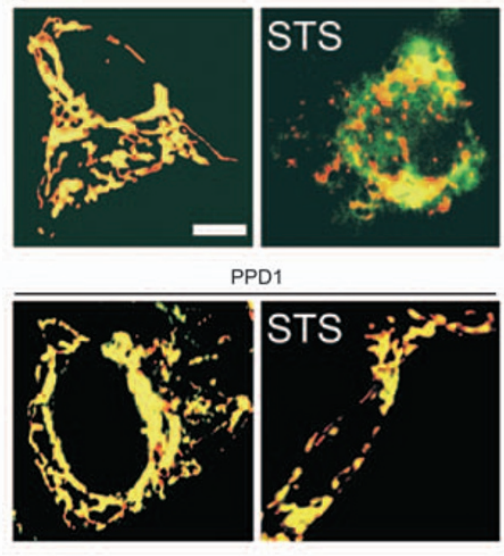

d
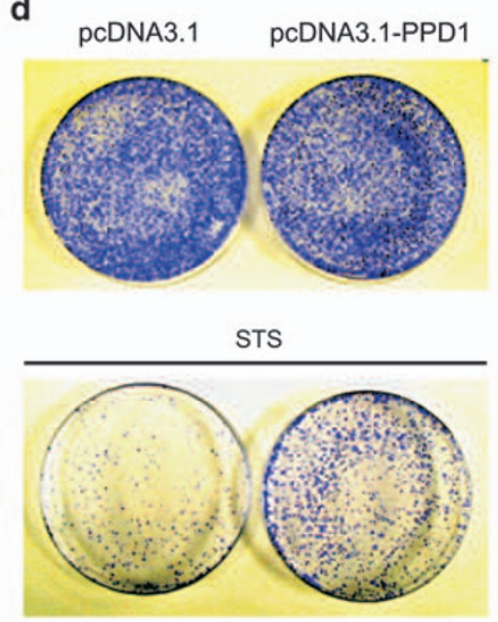
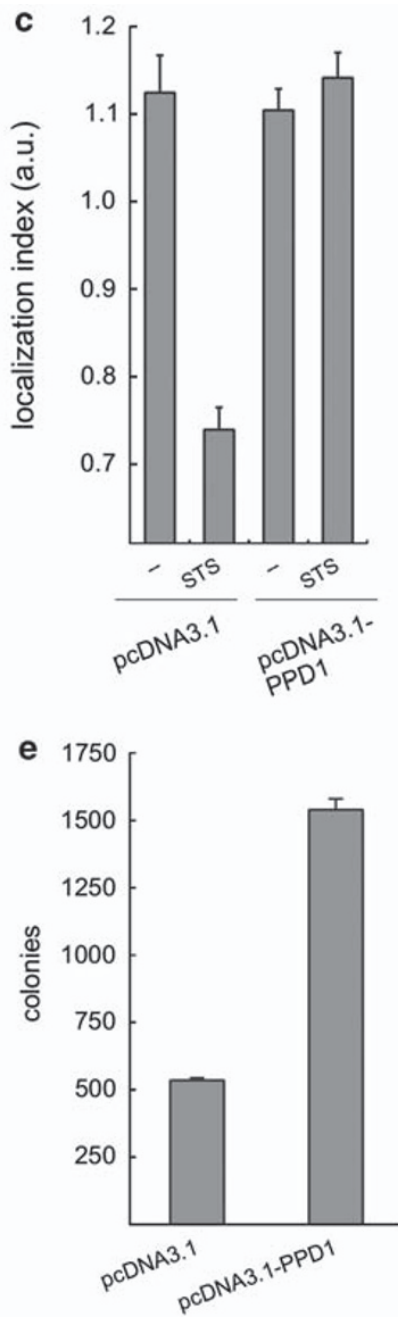

Figure 5 PPD1 acts at mitochondria to block apoptosis. (a) Representative frames acquired at indicated times from real-time confocal imaging of HeLa cells treated with staurosporine $(2 \mu \mathrm{M}) 24 \mathrm{~h}$ after cotransfection with mtRFP and the indicated plasmid. Bar, $20 \mu \mathrm{m}$. Time to fragmentation was $260 \pm 16.37$ min in control versus $347 \pm 10.15 \mathrm{in}$ PPD1 expressing cells $(n=3$ independent experiments, \pm S.E.). (b) Representative images of subcellular cytochrome $c$ distribution. HeLa cells were cotransfected with mtRFP (red) and the indicated plasmid. Where indicated, cells were treated for $2 \mathrm{~h}$ with staurosporine (2 $\mu \mathrm{M})$, fixed, and immunostained for cytochrome $c$ (green). Bar, $20 \mu \mathrm{m}$. (c) Localization index of cytochrome $c$. Experiments were performed as in (b). Data represent mean \pm S.E. of three independent experiments (d) Clonogenic survival assay. HeLa cells cotransfected with peGFP and the indicated plasmid were treated as indicated (staurosporine $2 \mu \mathrm{M}, 5 \mathrm{~h}$ ), sorted for GFP positivity by flow cytometry, and plated. After 12 days, colonies were visualized by staining with $0.1 \%$ crystal violet in $20 \%$ ethanol. (e) Quantitative analysis of the effect of PPD1 on clonogenic survival of STS-treated cells. Experiments were exactly as in (d) Data represent mean \pm S.E. of three different experiments

extend our analysis to investigate whether PPD1 displayed any antiapoptotic potential. Interestingly, PPD1 prevents release of cytochrome $c$ induced by staurosporine and delays apoptosis by a number of intrinsic stimuli that trigger death by different mechanisms (staurosporine, etoposide, $\mathrm{H}_{2} \mathrm{O}_{2}$, and thapsigargin). Our earlier analysis revealed that killing by these stimuli is complete only when levels of $\mathrm{Ca}^{2+}$ are adequate. ${ }^{26}$ This was for us a further suggestion that $\mathrm{Ca}^{2+}$. controlled pathway, such as the calcineurin one, participates in killing by these stimuli. However, whether PPD1 simply slowed down the kinetics of death, or really prevented activation of the post-mitochondrial cascade was unclear. Notably, PPD1 allows clonogenic survival of cells treated with staurosporine, an indication that cells can still proliferate and that caspases are not activated in a majority of cells expressing PPD1. Such a powerful effect could be supported by the pleiotropic nature of a calcineurin inhibitor like PPD1. Conversely, our genetic analysis revealed that the mode of action of PPD1 is rather specific. Reed and coworkers showed that $\mathrm{Ca}^{2+}$-mobilizing death stimuli induce activation of calcineurin. This in turn dephosphorylates the proapoptotic $\mathrm{BH} 3-$ only molecule BAD, promotes its recruitment on mitochondria, triggering permeabilization of the outer membrane and cell death. ${ }^{29}$ On the other hand, PPD1 is equally antiapoptotic despite the lack of BAD. As expected, deletion of Bad had an inhibitory effect on killing by staurosporine, but expression of PPD1 in $\mathrm{Bad}^{-1-}$ cells protected them as well as it did in their wild-type counterparts. Thus, PPD1 does not inhibit death through an effect on calcineurin-mediated dephosphorylation of BAD. In cells expressing a dominant-negative 

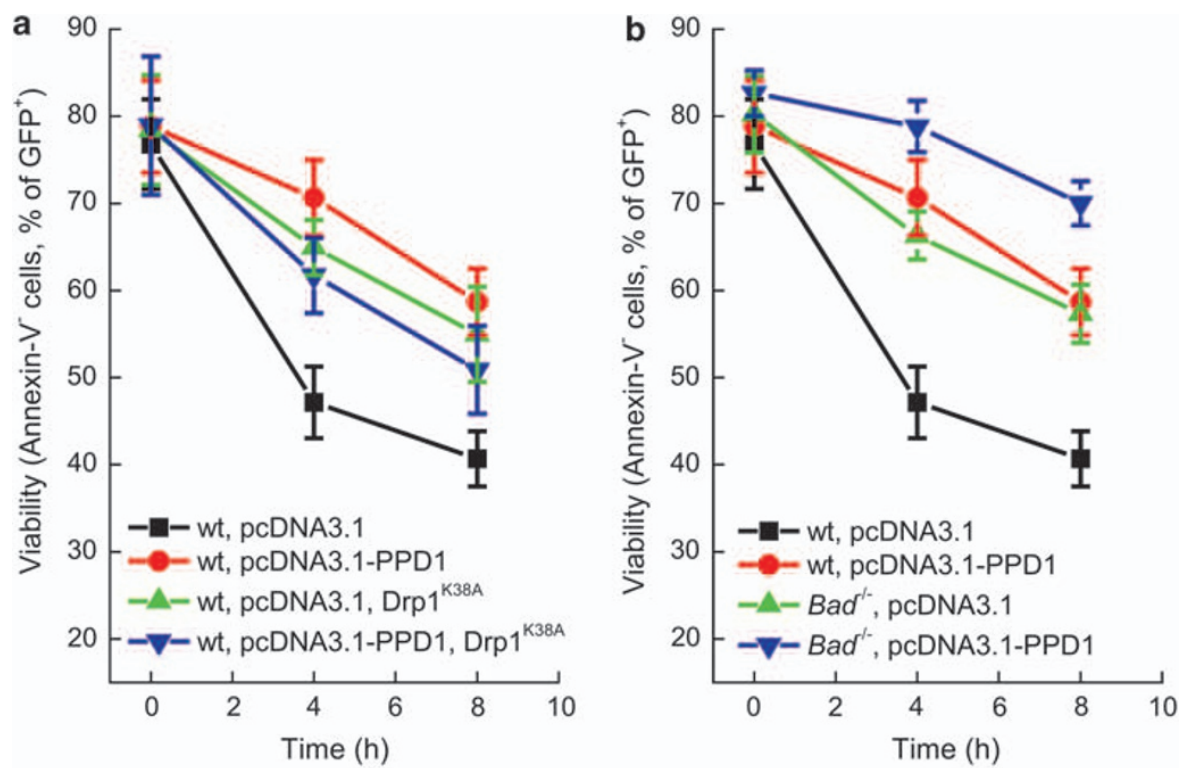

Figure 6 PPD1 blocks apoptosis by inhibiting Drp1. (a) MEFs cotransfected with pEGFP and the indicated plasmids were treated with staurosporine (2 $\mu$ M) for the indicated times and viability was determined by flow cytometry in the GFP-positive population. Data represent mean \pm S.E. of six independent experiments. (b) MEFs of the indicated genotype were cotransfected with pEGFP and the indicated plasmids and treated with staurosporine $(2 \mu \mathrm{M})$ for the indicated time. Viability was determined as in (a). Data are mean \pm S.E. of six independent experiments

Drp1, however, PPD1 has no further inhibitory effect, substantiating that it impinges on Drp1 to block apoptosis.

Our results further support the view that Drp1 and mitochondrial fragmentation participate in apoptosis. Indeed, earlier works showed that Drp1 GTPase activity participated in cell death, as both a Drp1 mutant defective in GTPase activity because of the substitution of a lysine in the catalytic site for an alanine, K38ADrp $1,{ }^{4}$ as well as a chemical inhibitor of the hydrolytic activity of the protein, mdivi- $1,{ }^{21}$ were successful in delaying cell death. The precise mechanism by which inhibition of Drp1 affects progression of cell death remains to be elucidated. Expression of PPD1 does not affect Bax translocation, or Smac/DIABLO release, whereas it blunts that of cytochrome $c$. This is in accordance with the finding that Smac/DIABLO and AIF are released even in the absence of Drp1, whereas cytochrome $c$ is retained in the organelle. ${ }^{18,20,31}$ As a matter of fact, cytochrome $c$ is highly compartmentalized inside mitochondria, given its preferential localization inside the cristae. ${ }^{6,32}$ This raises the possibility that the effects of blocking Drp1 in apoptosis are not a consequence of preservation of mitochondrial morphology, but of ultrastructure. This is confirmed by the finding that remodeling of the cristae, which mobilizes cytochrome $c$, is triggered by Drp1 and blocked by a dominant-negative mutant of this dynamin-related protein. ${ }^{19}$ This scenario might be even more complicated as ablation of Drp1 causes compensatory changes in the cleavage of the inner membrane Opa $1^{33}$ that controls cristae remodeling during apoptosis. ${ }^{9}$ In this respect, PPD1 and blockage of calcineurin, that is the Drp1 branch of apoptosis, could be an appealing mechanism to investigate, and in perspective to be pharmacologically attacked: it does not interfere with levels, but with mitochondrial translocation of Drp1.
Increasing evidence indicates that mitochondrial fragmentation is a hallmark of several diseases associated with increased apoptosis, from Alzheimer's ${ }^{34}$ to Parkinson's ${ }^{35}$ disease. In addition, calcineurin hyperactivity has been reported to occur during cell death by a variety of intrinsic stimuli (etoposide, ${ }^{36}$ thapsigargin ${ }^{37}$ ), as well as in relevant pathological conditions such as Parkinson's, Alzheimer's, and Huntington's diseases. Thus, the checkpoint controlled by calcineurin could serve as a potential therapeutic target for compounds or small peptides that mimic the action of PPD1.

\section{Materials and Methods}

Subcellular fractionation, immunoprecipitation, and immunoblotting. Subcellular fractionation was performed as described in Frezza et $\mathrm{al}^{38}$ For immunoprecipitation experiments, cells were lysed in CPBS buffer $(6 \mathrm{mM}$ CHAPS in PBS, pH 7.4). Lysates were incubated with anti-Drp1 antibody (1:50, $14 \mathrm{~h}, 4^{\circ} \mathrm{C}$ ) and the protein-antibody complex was precipitated by centrifugation after incubation with protein G-coated magnetic beads (Dynal, $2 \mathrm{~h}, 4^{\circ} \mathrm{C}$ ). The immunoprecipitated material was washed twice in CPBS and resuspended in SDS-PAGE loading buffer (NuPAGE), boiled, and loaded on 4-12\% gels (NuPAGE). For immunoblotting, proteins were transferred onto polyvinylidene fluoride (Millipore, Billerica, MA, USA) membranes and probed with the following antibodies: $\alpha$-Drp1 (BD Biosciences, San Jose, CA, USA; $1: 2000) ; ~ \alpha$-cyclophilinA (Upstate, Billerica, MA, USA; 1:2000); isotype-matched, horseradish peroxidaseconjugated secondary antibodies (Amersham, Freiburg, Germany) were used, followed by detection by chemiluminescence (Amersham).

Calcineurin activity assay. Cells grown on 24-mm wells incubated in HBSS buffer were treated as indicated, washed several times with TBS buffer $(10 \mathrm{mM}$ Tris-Cl, pH 7.4, $150 \mathrm{mM} \mathrm{NaCl}$ ), resuspended in TBS, and lysed in CTBS buffer (6 mM CHAPS in TBS, pH 7.4). Calcineurin activity was determined using an in vitro assay kit and following manufacturer's instructions (Calbiochem, Darmstadt, Germany). For comparative reasons, basal calcineurin activity in untreated cells was set to $100 \%$. 
Imaging. Imaging of mitochondrial membrane potential in HeLa cells was performed as described earlier. ${ }^{28}$ Data are graphed as percentage of the initial value for comparative reasons. For confocal imaging, $10^{5}$ cells seeded onto $24 \mathrm{~mm}$ round glass coverslips were transfected as indicated and after $24 \mathrm{~h}$ were incubated in HBSS supplemented with $10 \mathrm{mM}$ Hepes and placed on the stage of a Nikon Eclipse TE300 inverted microscope equipped with a spinning-disk PerkinElmer Ultraview $\mathrm{LCl}$ confocal system, a piezoelectric $z$ axis motorized stage (Pifoc, Physik Instrumente), and a Orca ER 12-bit CCD camera (Hamamatsu Photonics). Cells expressing mtRFP were excited using the 568-nm line of the He-Ne laser (PerkinElmer, Waltham, MA, USA) with exposure times of $100 \mathrm{~ms}$ using a $60 \times 1.4$ NA Plan Apo objective (Nikon). In time-course experiments, images were acquired every $10 \mathrm{~s}$ for $40 \mathrm{~min}$. Quantitative analysis of mitochondrial shape changes was performed by evaluating the time at which cells displayed fragmented mitochondria after addition of the inducer. Organelles were classified as fragmented when $50 \%$ of the total cellular mitochondria displayed a major axis $<5 \mu \mathrm{m}$.

For cytochrome c immunolocalization, cells grown on coverslips were transfected with mtRFP and after $24 \mathrm{~h}$ incubated as detailed. Immunostaining for cytochrome $c$ was performed as described in Scorrano et al. ${ }^{26}$ with an anti-cytochrome $c$ antibody (BD Biosciences, 1:200). For cytochrome $c$ and mtRFP detection, green and red channel images were acquired simultaneously using two separate color channels on the detector assembly of a Nikon Eclipse E600 microscope equipped with a Biorad MRC-1024 laser scanning confocal imaging system. The localization index was calculated as described in Petronilli et al. ${ }^{28}$

Analysis of cell death. A total of $1 \times 10^{5} \mathrm{HeLa}$ cells or MEFs grown in 12-well plates were cotransfected with pEGFP and the indicated vectors. At the time points indicated, cells were treated as described and stained with Annexin-V-Alexa568 (Roche, Basel, Switzerland) according to manufacturer's protocol. Apoptosis was measured by flow cytometry (FACSCalibur) as the percentage of Annexin-Vpositive events in the GFP-positive population.

Clonogenic assay. A total of $1 \times 10^{6} \mathrm{HeLa}$ cells seeded on $10 \mathrm{~cm}$ Petri dishes were cotransfected with pEGFP and PPD1 or empty vector. After $24 \mathrm{~h}$, cells were sorted by FACS and 50000 GFP positive were treated with $2 \mu \mathrm{M}$ staurosporine in DMSO or DMSO alone. After $5 \mathrm{~h}$, cells were washed with PBS, trypsinized, resuspended in complete DMEM (DMEM, Invitrogen (Basel, Switzerland), supplemented with $10 \%$ fetal bovine serum, $2 \mathrm{mM}$ glutamine, $1 \%$ non-essential amino-acid mix (Gibco, Basel, Switzerland), $1 \mathrm{mM}$ penicillin/streptomycin) and plated on $10 \mathrm{~cm}$ Petri dishes containing $15 \mathrm{ml}$ of DMEM supplemented with $2 \mathrm{mM}$ glutamine, $1 \mathrm{mM}$ penicillin/streptomycin, $20 \%$ fetal bovine serum and $0.3 \%$ Bacto Agar (Invitrogen). Plates were incubated at $37^{\circ} \mathrm{C}$ in a fully humidified atmosphere of $95 \%$ air and $5 \% \mathrm{CO}_{2}$. After 12 days, colonies were visualized by $0.1 \%$ crystal violet in $20 \%$ ethanol.

\section{Conflict of interest}

The authors declare no conflict of interest.

Acknowledgements. We are thankful to Dr. Jack-Michel Renoir and Dr. Christine Radanyi for providing a vector for PPD1 expression and Dr. Nika Danial (Boston, USA) for providing $\mathrm{Bad}^{-1-}$ MEFs cells as well as Dr. C Blackstone and Dr. C Chang (Bethesda, USA) for the anti-phosphoSer637Drp1 antibody. This work was supported by SNF, Telethon Italy, Oncosuisse.

1. Wang $X$ (2001) The expanding role of mitochondria in apoptosis. Genes Dev 15 2922-2933.

2. Danial NN, Korsmeyer SJ (2004) Cell death: critical control points. Cell 116: 205-219.

3. Lovell JF, Billen LP, Bindner S, Shamas-Din A, Fradin C, Leber B et al. (2008) Membrane binding by tBid initiates an ordered series of events culminating in membrane permeabilization by Bax. Cell 135: 1074-1084

4. Frank S, Gaume B, Bergmann-Leitner ES, Leitner WW, Robert EG, Catez F et al. (2001) The role of dynamin-related protein 1, a mediator of mitochondrial fission, in apoptosis. Dev Cell 1: 515-525.

5. Martinou I, Desagher S, Eskes R, Antonsson B, Andre E, Fakan S et al. (1999) The release of cytochrome $c$ from mitochondria during apoptosis of NGF-deprived sympathetic neurons is a reversible event. $J$ Cell Biol 144: 883-889.
6. Scorrano L, Ashiya M, Buttle K, Weiler S, Oakes SA, Mannella CA et al. (2002) A distinct pathway remodels mitochondrial cristae and mobilizes cytochrome $c$ during apoptosis. Dev Cell 2: 55-67.

7. Wasilewski M, Scorrano L (2009) The changing shape of mitochondrial apoptosis. Trends Endocrinol Metab 20: 287-294.

8. Cipolat S, Rudka T, Hartmann D, Costa V, Serneels L, Craessaerts K et al. (2006) Mitochondrial rhomboid PARL regulates cytochrome $c$ release during apoptosis via OPA1dependent cristae remodeling. Cell 126: 163-175.

9. Frezza C, Cipolat S, Martins dB, Micaroni M, Beznoussenko GV, Rudka T et al. (2006) OPA1 controls apoptotic cristae remodeling independently from mitochondrial fusion. Cell 126: 177-189.

10. de Brito OM, Scorrano L (2008) Mitofusin 2 tethers endoplasmic reticulum to mitochondria. Nature 456: 605-610

11. Smirnova E, Griparic L, Shurland DL, van der Bliek AM (2001) Dynamin-related protein Drp1 is required for mitochondrial division in mammalian cells. Mol Biol Cell 12: 2245-2256.

12. Yoon Y, Krueger EW, Oswald BJ, McNiven MA (2003) The mitochondrial protein hFis1 regulates mitochondrial fission in mammalian cells through an interaction with the dynaminlike protein DLP1. Mol Cell Biol 23: 5409-5420.

13. Cereghetti GM, Scorrano L (2006) The many shapes of mitochondrial death. Oncogene 25 : 4717-4724.

14. Cereghetti GM, Stangherlin A, Martins dB, Chang CR, Blackstone C, Bernardi P et al. (2008) Dephosphorylation by calcineurin regulates translocation of Drp1 to mitochondria. Proc Natl Acad Sci USA 105: 15803-15808.

15. Karbowski M, Lee YJ, Gaume B, Jeong SY, Frank S, Nechushtan A et al. (2002) Spatial and temporal association of Bax with mitochondrial fission sites, Drp1, and Mfn2 during apoptosis. J Cell Biol 159: 931-938.

16. Arnoult D, Rismanchi N, Grodet A, Roberts RG, Seeburg DP, Estaquier J et al. (2005) Bax Bak-dependent release of DDP/TIMM8a promotes Drp1-mediated mitochondrial fission and mitoptosis during programmed cell death. Curr Biol 15: 2112-2118.

17. Yuan H, Gerencser AA, Liot G, Lipton SA, Ellisman M, Perkins GA et al. (2007) Mitochondrial fission is an upstream and required event for bax foci formation in response to nitric oxide in cortical neurons. Cell Death Differ 14: 462-471.

18. Parone PA, James DI, Da Cruz S, Mattenberger Y, Donze O, Barja F et al. (2006) Inhibiting the mitochondrial fission machinery does not prevent Bax/Bak-dependent apoptosis. Mol Cell Biol 26: 7397-7408.

19. Germain M, Mathai JP, McBride HM, Shore GC (2005) Endoplasmic reticulum BIK initiates DRP1-regulated remodelling of mitochondrial cristae during apoptosis. EMBO J 24: $1546-1556$.

20. Ishihara N, Nomura M, Jofuku A, Kato H, Suzuki SO, Masuda K et al. (2009) Mitochondrial fission factor Drp1 is essential for embryonic development and synapse formation in mice. Nat Cell Biol 11: 958-966.

21. Cassidy-Stone A, Chipuk JE, Ingerman E, Song C, Yoo C, Kuwana T et al. (2008) Chemical inhibition of the mitochondrial division dynamin reveals its role in Bax/Bak-dependent mitochondrial outer membrane permeabilization. Dev Cell 14: 193-204.

22. Sheridan C, Delivani $P$, Cullen SP, Martin SJ (2008) Bax- or Bak-induced mitochondrial fission can be uncoupled from cytochrome $C$ release. Mol Cell 31: 570-585.

23. Galigniana MD, Morishima Y, Gallay PA, Pratt WB (2004) Cyclophilin-A is bound through its peptidylprolyl isomerase domain to the cytoplasmic dynein motor protein complex. J Biol Chem 279: 55754-55759.

24. Varadi A, Johnson-Cadwell LI, Cirulli V, Yoon Y, Allan VJ, Rutter GA (2004) Cytoplasmic dynein regulates the subcellular distribution of mitochondria by controlling the recruitment of the fission factor dynamin-related protein-1. J Cell Sci 117: 4389-4400.

25. Galigniana MD, Radanyi C, Renoir JM, Housley PR, Pratt WB (2001) Evidence that the peptidylprolyl isomerase domain of the hsp90-binding immunophilin FKBP52 is involved in both dynein interaction and glucocorticoid receptor movement to the nucleus. J Biol Chem 276: 14884-14889.

26. Scorrano L, Oakes SA, Opferman JT, Cheng EH, Sorcinelli MD, Pozzan T et al. (2003) $\mathrm{BAX}$ and $\mathrm{BAK}$ regulation of endoplasmic reticulum $\mathrm{Ca2}+$ : a control point for apoptosis. Science 300: 135-139.

27. Lee YJ, Jeong SY, Karbowski M, Smith CL, Youle RJ (2004) Roles of the mammalian mitochondrial fission and fusion mediators Fis1, Drp1, and Opa1 in apoptosis. Mol Biol Cell 15: 5001-5011.

28. Petronilli V, Penzo D, Scorrano L, Bernardi P, Di Lisa F (2001) The mitochondria permeability transition, release of cytochrome $C$ and cell death. Correlation with the duration of pore openings in situ. J Biol Chem 276: 12030-12034.

29. Wang HG, Pathan N, Ethell IM, Krajewski S, Yamaguchi Y, Shibasaki F et al. (1999) $\mathrm{Ca2+-induced} \mathrm{apoptosis} \mathrm{through} \mathrm{calcineurin} \mathrm{dephosphorylation} \mathrm{of} \mathrm{BAD.} \mathrm{Science} 284$ : 339-343.

30. Puthalakath H, Huang DC, O'Reilly LA, King SM, Strasser A (1999) The proapoptotic activity of the Bcl-2 family member Bim is regulated by interaction with the dynein motor complex. Mol Cell 3: 287-296.

31. Estaquier J, Arnoult D (2007) Inhibiting Drp1-mediated mitochondrial fission selectively prevents the release of cytochrome $c$ during apoptosis. Cell Death Differ 14: 1086-1094.

32. Bernardi P Azzone GF (1981) Cytochrome $c$ as an electron shuttle between the outer and inner mitochondrial membranes. J Biol Chem 256: 7187-7192. 
33. Mopert K, Hajek P, Frank S, Chen C, Kaufmann J, Santel A (2009) Loss of Drp1 function alters OPA1 processing and changes mitochondrial membrane organization. Exp Cell Res 315: 2165-2180.

34. Wang X, Su B, Lee HG, Li X, Perry G, Smith MA et al. (2009) Impaired balance of mitochondrial fission and fusion in Alzheimer's disease. J Neurosci 29: 9090-9103.

35. Lutz AK, Exner N, Fett ME, Schlehe JS, Kloos K, Lammermann K et al. (2009) Loss of parkin or PINK1 function increases Drp1-dependent mitochondrial fragmentation. J Biol Chem 284: 22938-22951.
36. Rivera A, Maxwell SA (2005) The p53-induced gene-6 (proline oxidase) mediates apoptosis through a calcineurin-dependent pathway. J Biol Chem 280 29346-29354.

37. Muramoto M, Yamazaki T, Morikawa N, Okitsu O, Nagashima T, Oe T et al. (2005) Protective effect of FK506 against apoptosis of SH-SY5Y cells correlates with regulation of the serum inducible kinase gene. Biochem Pharmacol 69: 1473-1481.

38. Frezza C, Cipolat S, Scorrano $L$ (2007) Organelle isolation: functional mitochondria from mouse liver, muscle and cultured fibroblasts. Nat Protoc 2: 287-295.

Supplementary Information accompanies the paper on Cell Death and Differentiation website (http://www.nature.com/cdd) 